\title{
Blind Authentication Using Periodic Properties of Interpolation
}

\author{
Babak Mahdian*, Stanislav Saic
}

\begin{abstract}
In this paper we analyze and analytically describe the specific statistical changes brought into the covariance structure of signal by the interpolation process. We show that interpolated signals and their derivatives contain specific detectable periodic properties. Based on this, we propose a blind, efficient and automatic method capable of finding traces of resampling and interpolation. The proposed method can be very useful in many areas, especially in image security and authentication. For instance, when two or more images are spliced together, to create high quality and consistent image forgeries, almost always geometric transformations such as scaling, rotation or skewing are needed. These procedures are typically based on a resampling and interpolation step. By having a method capable of detecting the traces of resampling, we can significantly reduce the successful usage of such forgeries. Among other points, the presented method is also very useful in estimation of the geometric transformations factors.
\end{abstract}

Index Terms-Image forensics, digital forgery, image tampering, interpolation, resampling, interpolation detection, resampling detection.

\section{INTRODUCTION}

D ESPITE the importance, massive usage ${ }^{1}$ and history ${ }^{2}$ of interpolation, to our knowledge, there exist only a few published works concerned with the specific and detectable statistical changes brought into the signal by this process. In this paper we analytically describe specific periodic properties present in the covariance structure of interpolated signals and their $n$th derivatives. Without the detailed knowledge of how the statistics of the signal is changed by the interpolation process, applications based on statistical approaches working with resampled/interpolated signals or with their derivatives can yield miscalculations and unexpected results.

Furthermore, we propose a blind, efficient and automatic method capable of detecting the traces of resampling and interpolation. The method is based on a derivative operator and radon transformation. The knowledge of whether the given signal or some of its portions have been resampled can play an essential role in many fields, especially in image security and authentication. Without a doubt, image security and authentication are significant in many social areas and play a crucial role in people's lives. For instance, the trustworthiness

The authors are with the Institute of Information Theory and Automation, Academy of Sciences of the Czech Republic, Pod Vodárenskou věží 4, 18208 Prague 8, Czech Republic (e-mail: mahdian@utia.cas.cz; ssaic@utia.cas.cz).

This work has been partially supported by the Czech Science Foundation under the project No. GACR 102/08/0470.

${ }^{1}$ For instance, almost every image resizing or rotation operation requires an interpolation process (nearest neighbor, linear, cubic, etc.).

${ }^{2}$ Interpolation has a long history and probably started being used as early as $2000 \mathrm{BC}$ by ancient Babylonian mathematicians. For instance, it had an important role in astronomy which in those days was all about time-keeping and making predictions concerning astronomical events [1]. of photographs has an essential role in courtrooms, where they are used as evidence. Every day newspapers and magazines depend on digital images. Today, we face the problem of digital image forgeries even in scientific literature. As a consequence, it is obvious that we should pay special attention to the field of image authenticity. Therefore we believe in the wide possible use of the proposed method. Furthermore, the presented method is very useful in estimating the geometric transformations factors as well.

When two or more images are spliced together (for an example see Figure 1), to create high quality and consistent image forgeries, almost always geometric transformations such as scaling, rotation or skewing are needed. Geometric transformations typically require a resampling and interpolation step. Therefore, by having sophisticated resampling/interpolation detectors, altered images containing resampled portions can be easily identified and their successful usage significantly reduced.

Existing digital forgery detection methods are divided into active [2], [3], [4], and passive (blind) [5], [6], [7], [8], [9], [10] approaches. The passive (blind) approach is regarded as the new direction. In contrast to active approaches, passive approaches do not need any explicit prior information about the image. They work in the absence of any digital watermark or signature. Passive approaches have not yet been thoroughly researched. Different methods for identifying each type of forgery must be developed. Then by fusing the results from each analysis, a decisive conclusion may be drawn.

The rest of the paper is organized as follows. The next section summarizes previous published papers concerned with the topic of this paper. After this, some basic notations and definitions are given to build up the necessary mathematical background. Section 4 analyzes and analytically shows hidden periodic properties present in interpolated signals and in their derivatives. In section 5 we study periodic properties brought into the signal by concrete interpolation kernels. Section 6 propose a method capable of detecting the traces of affine transformation. The following section contains experiments which demonstrate the outcomes of the proposed method. In section 8 the main properties of the method and its results are discussed. The last section summarizes the work that has been done in this paper.

\section{RELATED WORK}

In [11], A. C. Gallagher in an effort to detect interpolation in digitally zoomed images has found that linear and cubic interpolated signals introduce periodicity in variance function of their second order derivative. This periodicity is simply 

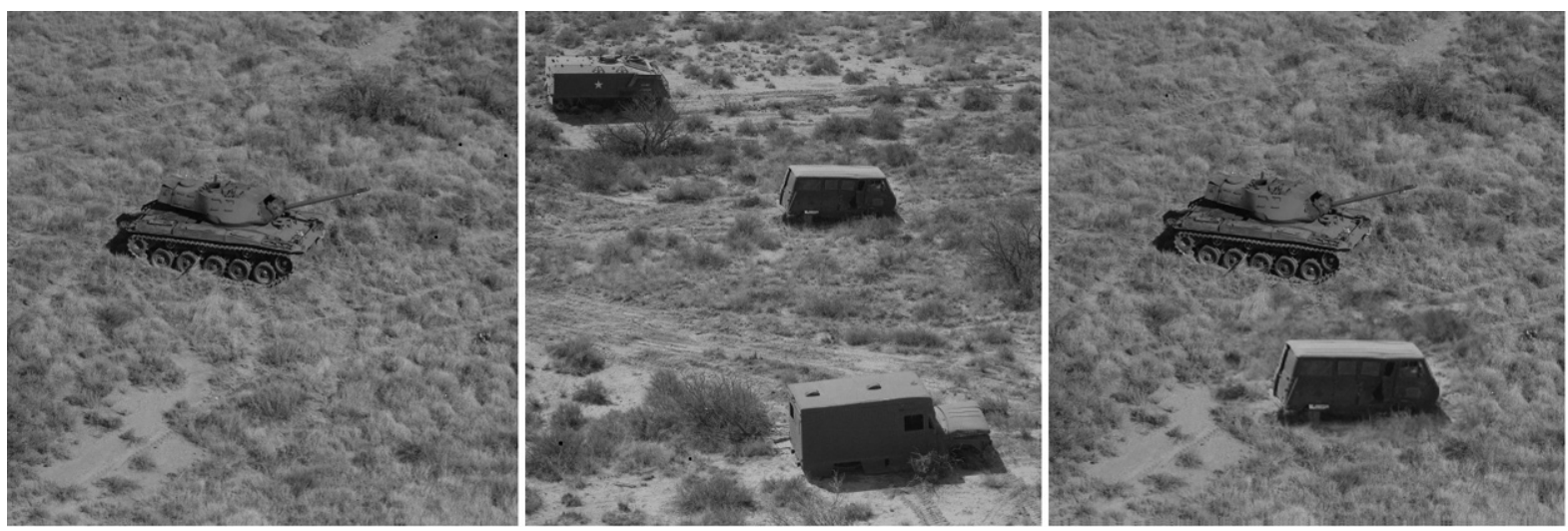

Fig. 1. An example of image forgery based on resampling and interpolation. Shown from left to right are: source image A, source image B and tampered image. The tampered image has been created by splicing source image A with a resized part of source image B. This part has been resized by scaling factor 1.42 using the bicubic interpolation

investigated by computing the DFT of an averaged signal obtained from the second derivative of the investigated signal. Furthermore, based on the fact that the mentioned periodicity is directly related to the resampling rate, the author easily estimates the resampling rate. The major weakness of the method is that it cannot be applied to rotated or skewed images.

Another work concerned with the detection of resampling and interpolation has been proposed by S. Prasad and K. R. Ramakrishnan [10]. Similar to [11], the authors have noticed that the second derivative of an interpolated signal produces detectable periodic properties. The periodicity is simply detected in the frequency domain by analyzing a binary signal obtained by zero crossings of the second derivative of the interpolated signal. The major weaknesses of this method are similar to [11].

In an interesting work [7], A. C. Popescu and H. Farid have analyzed the imperceptible specific correlations brought into the resampled signal by the interpolation step. Their method is based on the fact that in a resampled signal it is possible to find a set of periodic samples that are correlated in the same way as their neighbors. The core of the method is an Expectation/Maximization (EM) algorithm. The main output of the method is a probability map containing periodic patterns if the investigated signal has been resampled.

As it is apparent, the subject of this paper has not been studied in detail. In this work, we study and analytically describe the periodic properties of the covariance structure of interpolated signals and their derivatives. Using the theory we bring the main contribution of this paper which is a fast, blind and efficient method capable of detecting traces of arbitrary affine transformation. The method can be also used for estimating the scaling factors or rotation angles as well as skewing factors. The core of our method is a radon transformation applied to the derivative of the investigated signal. In this paper, among other points, we extend and generalize the theory introduced in [11]. We show that the specific periodicity is present in interpolated signals as well as in their derivatives. We briefly extend the theory for two-dimensional cases as well. Also we analyze and show periodic patterns of interpolation by an application of Taylor series to the interpolated signals. To summarize, the main novelties of the paper are the followings: the use of the radon transformation making possible the detection of rotation and skewing, the generalization of the theory introduced in [11] and an application of Taylor series to the interpolated signals showing a hidden periodicity.

Obtained results of the proposed method show that it is possible in a simple and fast way to find traces of general affine transformation when a low order interpolation polynomial has been used. To our knowledge, the only published method comparable with our method is [7]. In comparison to this work, our method works on a much easier principle and is more convenient and faster to implement and run. Another advantage of the proposed method compared to [7] is that it does not need any initialization parameters which can strongly affect obtained outcomes.

\section{BASIC Notations ANd PRELIMINARIES}

First, a proper mathematical model simulating the acquisition system is required. Periodic properties of interpolation can be effectively studied by using the following simple, linear and stochastic model and assumptions:

$$
f(x)=(u * h)(x)+n(x)
$$

where $f, u, h, *$, and $n$ are the measured image, original image, system PSF, convolution operator, and random variable representing the influence of noise sources statistically independent from the signal part of the image. We assume that $E\{n(x)\}=0$. If we consider the first part of (1) to be deterministic, the covariance of (1) can be shown to be $R_{f}\left(x_{1}, x_{2}\right)=\operatorname{Cov}\left\{f\left(x_{1}\right), f\left(x_{2}\right)\right\}=E\left\{\left(f\left(x_{1}\right)-\right.\right.$ $\left.\left.\bar{f}\left(x_{1}\right)\right)\left(f\left(x_{2}\right)-\bar{f}\left(x_{2}\right)\right)\right\}=\operatorname{Cov}\left\{n\left(x_{1}\right), n\left(x_{2}\right)\right\}=R_{n}\left(x_{1}, x_{2}\right)$, where $R_{f}$ is the covariance matrix of measured image $f(x)$, and $R_{n}$ is the covariance of random process $n(x)$.

We will denote by $f_{k}$ a discrete signal representing the samples of $f(x)$ at the locations $k \Delta_{x}, f_{k}=f\left(k \Delta_{x}\right)$, where $\Delta_{x} \in \mathcal{R}^{+}$, is the sampling step and $k \in \mathcal{N}_{0}$.

For the sake of simplicity we introduce the operator $\mathcal{D}^{n}\{\bullet\}$, $n \in \mathcal{N}_{0}$, which is defined in the following way: $\mathcal{D}^{n}\{f\}(x)=$ 


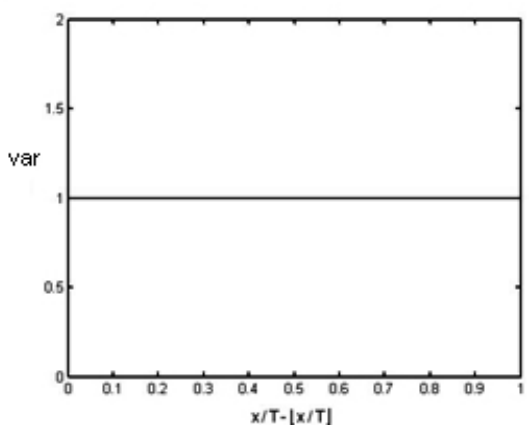

Fig. 2. The periodic variance function computed via Equation (3) for the nearest neighbor kernel.

$f(x)$ for $n=0$ and $\mathcal{D}^{n}\{f\}(x)=\frac{\partial^{n} f(x)}{\partial x^{n}}$ for $n \in \mathcal{N}$. In other words, $\mathcal{D}^{0}\{f\}(x)$ is identical to $f(x)$ and $\mathcal{D}^{n}\{f\}(x)$, where $n>0$, is the $n$th derivative of $f(x)$. In discrete signals derivative is typically approximated by computing the finite difference between adjacent samples.

\section{Hidden Periodicity of Interpolated Signals}

There are two basic steps in geometric transformations. In the first step a spatial transformation of the physical rearrangement of pixels in the image is done. Coordinate transformation is described by a transformation function, $T$, which maps the coordinates of the input image pixel to the point in the output image (or vice versa):

$$
x^{\prime}=T_{x}(x, y) \quad y^{\prime}=T_{y}(x, y)
$$

As mentioned previously, the most used geometric operations in order to create a consistent forgeries are scaling, rotation, skewing or any arbitrary combination. Hence, we focus our work on analyzing and detecting the traces of affine transformation. General affine transformation can be described by following equations:

$$
x^{\prime}=a_{0}+a_{1} x+a_{2} y \quad y^{\prime}=b_{0}+b_{1} x+b_{2} y
$$

The second step in geometric transformations is called the interpolation step. Here pixels intensity values of the transformed image are assigned using a constructed low-pass interpolation filter, $w$. To compute signal values at arbitrary locations, as the word interpolation signifies ${ }^{3}$ discrete samples of $f_{k}$ are multiplied with the proper filter weights when convolving them with $w$.

Following the sampling theory, if the Nyquist criterion is satisfied, the spectrum $F(\omega)$ do not overlap in the Fourier domain. The original signal $f(x)$ can be reconstructed perfectly from its samples $f_{k}$ using the optimal sinc interpolator. The sinc function is hard to implement in practice because of its infinite extent. Thus, many different simpler interpolation kernels of bounded support have been investigated and proposed so far [12], [13], [14]. We will be concerned mainly with following low-order piecewise local polynomials: nearestneighbor, linear, cubic and truncated sinc. These polynomials

\footnotetext{
${ }^{3}$ The word "interpolation" originates from the Latin word "inter", meaning "between", and verb "polare", meaning "to polish" [1].
}

are used extensively because of their simplicity and implementation unassuming properties.

Combining the derivative theorem with the convolution theorem leads to the conclusion that by convolution of $f_{k}$ with a derivative kernel $\mathcal{D}^{n}\{w\}$, it is possible to reconstruct the $n$th derivative of $f(x)$. We denote the result of interpolation operation by $f^{w}(x)$, respectively by $\mathcal{D}\left\{f^{w}\right\}(x)$. Formally,

$$
\begin{aligned}
f^{w}(x) & =\sum_{k=-\infty}^{\infty} f_{k} w\left(\frac{x}{\Delta_{x}}-k\right) \\
\mathcal{D}^{n}\left\{f^{w}\right\}(x) & =\mathcal{D}^{n}\left\{\sum_{k=-\infty}^{\infty} f_{k} w\left(\frac{x}{\Delta_{x}}-k\right)\right\}=\sum_{k=-\infty}^{\infty} f_{k} \mathcal{D}^{n}\{w\}\left(\frac{x}{\Delta_{x}}-k\right)
\end{aligned}
$$

As pointed out in [15], it is easy to show that the covariance function of an interpolated image or its derivative is given by:

$$
R_{\mathcal{D}^{n}\left\{f^{w}\right\}}(x, x+\xi)=\sum_{k_{1}=-\infty}^{\infty} \sum_{k_{2}=-\infty}^{\infty} \mathcal{D}^{n}\{w\}\left(\frac{x}{\Delta_{x}}-k_{1}\right) \mathcal{D}^{n}\{w\}\left(\frac{x+\xi}{\Delta_{x}}-\right.
$$

If we assume constant variance random process, then the variance of $\mathcal{D}^{n}\left\{f^{w}\right\}, \operatorname{var}\left\{\mathcal{D}^{n}\left\{f^{w}\right\}(x)\right\}$, as a function of the position $x$ can be represented in the following way:

$\operatorname{var}\left\{\mathcal{D}^{n}\left\{f^{w}\right\}(x)\right\}=R_{\mathcal{D}^{n}\left\{f^{w}\right\}}(x, x)=\sigma^{2} \sum_{k=-\infty}^{\infty} \mathcal{D}^{n}\{w\}\left(\frac{x}{\Delta_{x}}-k\right)^{2}$

where $\sigma^{2}=R_{n}\left(k_{1}, k_{2}\right)$. This equation can be obtained if $R_{f}\left(k_{1}, k_{2}\right)$ has a short-range correlation [15]. Similarly, the covariance can be represented like:

$R_{\mathcal{D}^{n}\left\{f^{w}\right\}}(x, x+\xi)=\sigma^{2} \sum_{k=-\infty}^{\infty} \mathcal{D}^{n}\{w\}\left(\frac{x}{\Delta_{x}}-k\right) \mathcal{D}^{n}\{w\}\left(\frac{x+\xi}{\Delta_{x}}-k\right)$

Now, by assuming that $\vartheta$ is an integer, we can notice that

$\operatorname{var}\left\{\mathcal{D}^{n}\left\{f^{w}\right\}(x)\right\}=\operatorname{var}\left\{\mathcal{D}^{n}\left\{f^{w}\right\}\left(x+\vartheta \Delta_{x}\right)\right\}, \vartheta \in \mathcal{Z}$

Thus, $\operatorname{var}\left\{\mathcal{D}^{n}\left\{f^{w}\right\}(x)\right\}$ is periodic over $\mathrm{x}$ with period $\Delta_{x}$ (as aforementioned, $\Delta_{x}$ is the sampling step). We verify this in the following way:

$$
\begin{array}{r}
\operatorname{var}\left\{\mathcal{D}^{n}\left\{f^{w}\right\}\left(x+\vartheta \Delta_{x}\right)\right\}=\sigma^{2} \sum_{k=-\infty}^{\infty} \mathcal{D}^{n}\{w\}\left(\frac{x+\vartheta \Delta_{x}}{\Delta_{x}}-k\right)^{2} \\
=\sigma^{2} \sum_{k=-\infty}^{\infty} \mathcal{D}^{n}\{w\}\left(\frac{x}{\Delta_{x}}-(k-\vartheta)\right)^{2}=\operatorname{var}\left\{\mathcal{D}^{n}\left\{f^{w}\right\}(x)\right\}
\end{array}
$$

In other words we have shown that interpolation brings into the signal and their derivatives a specific periodicity. This periodicity is dependant on the interpolation kernel used. Several widely used interpolation kernels will be studied in the next section.

Similarly, it can be shown that the covariance of $f^{w}$, $R_{\mathcal{D}^{n}\left\{f^{w}\right\}}(x, x+\xi)$, is periodic as well. The periodicity is apparent for offset $\xi=\vartheta \Delta_{x}, \vartheta \in \mathcal{Z}$.

$$
R_{\mathcal{D}^{n}\left\{f^{w}\right\}}(x, x+\xi)=R_{\mathcal{D}^{n}\left\{f^{w}\right\}}\left(x, x+\vartheta \Delta_{x}\right), \vartheta \in \mathcal{Z}
$$

Before going on, it can be interesting to have a look on application of Taylor series on $\mathcal{D}^{n}\left\{f^{w}\right\}(x)$. By application of Taylor series, the hidden periodicity of the interpolation 

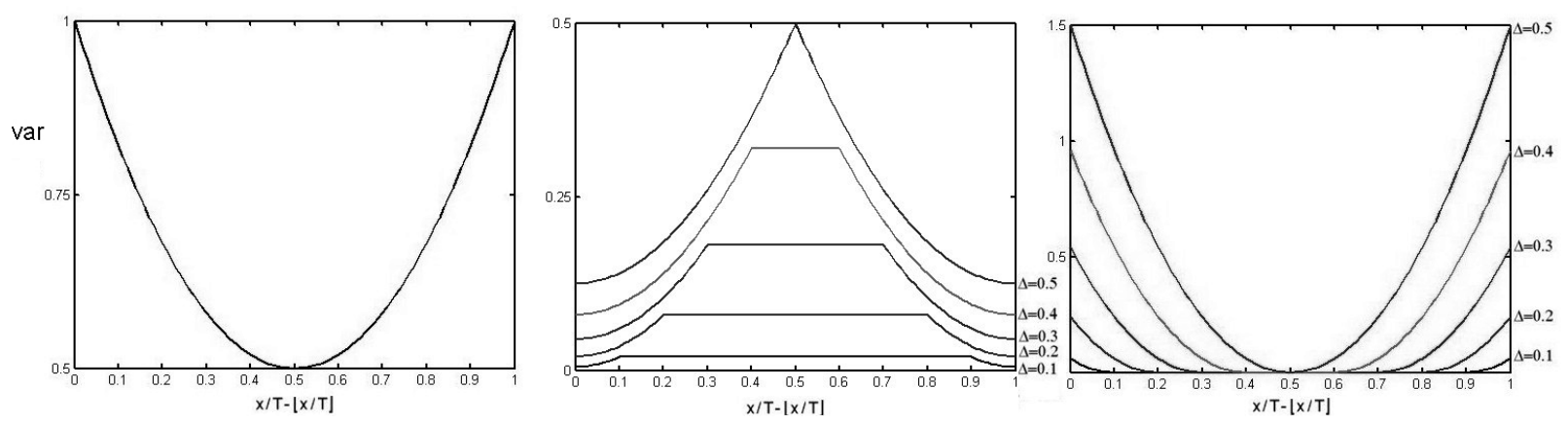

Fig. 3. The periodic variance of the linear, linear first and second derivative kernel computed via Equation (3).
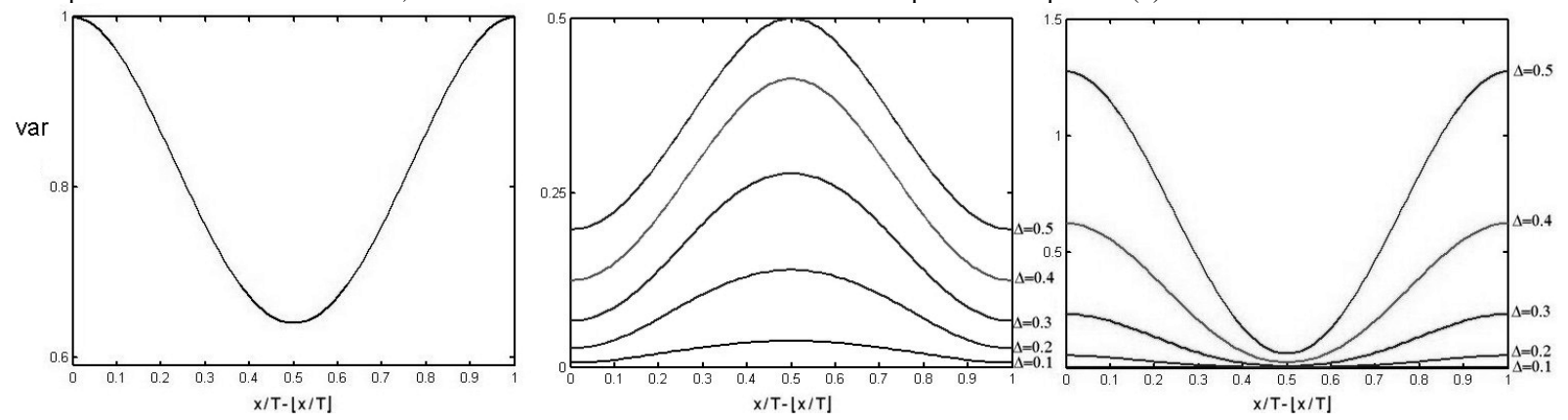

Fig. 4. The periodic variance of the cubic, cubic first and second derivative kernel computed via Equation (3).
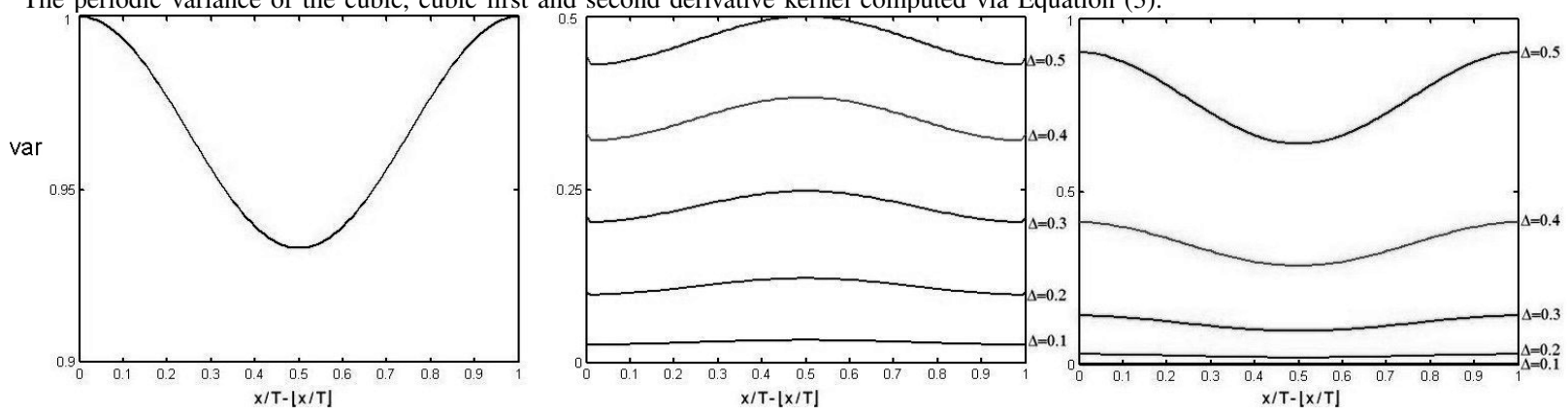

Fig. 5. The periodic variance of the truncated sinc, first and second derivative truncated sinc kernel $(N=6)$.

process can also be noticed and analyzed. By assuming that the first $(m+1)$ derivatives of $f(x)$ exist, we can rewrite Equation (2) as following:

$$
\begin{aligned}
& \mathcal{D}^{n}\left\{f^{w}\right\}(x)=\sum_{k=-\infty}^{\infty}\left\{\sum_{m=0}^{m} \frac{\mathcal{D}^{m}\{f\}(x)}{m !}\left(k \Delta_{x}-x\right)^{m}+R_{m+1}(x)\right. \\
& \text { By defining } \\
& \qquad \tilde{T}_{m}(x)=\sum_{k=-\infty}^{\infty} \frac{\left(k \Delta_{x}-x\right)^{m}}{m !} \mathcal{D}^{n}\{w\}\left(\frac{x}{\Delta_{x}}-k\right) \\
& \text { and } \\
& \tilde{R}_{m+1}\left(x, k \Delta_{x}\right)=\sum_{k=-\infty}^{\infty} R_{m+1}\left(x, k \Delta_{x}\right) \mathcal{D}^{n}\left\{w\left(\frac{x}{\Delta_{x}}-k\right)\right\}
\end{aligned}
$$

we can rewrite (5) as:

$$
\mathcal{D}^{n}\left\{f^{w}(x)\right\}=\sum_{m=0}^{m} \tilde{T}_{m}(x) \mathcal{D}^{n}\{f\}(x)+\tilde{R}_{m+1}\left(x, k \Delta_{x}\right)
$$

Now, by analyzing $\tilde{T}_{m}(x)$ we can notice that it is periodic with period $\Delta_{x}$ as well: 


\section{ReSAMPLED Signals}

As it is apparent from Equation (3) different interpolators change the structure of the signal in different ways. Figure 2 shows the resulting periodic variance function computed via Equation (3) with $\sigma=1$ for nearest neighbor interpolation. Nearest neighbor interpolator is a zero-degree kernel and the simplest of all piecewise, local polynomials. As it is shown the variance is a constant function. Note that derivatives of the nearest neighbor polynomial are zero. Hence, signals interpolated by this interpolator can be easily recognized by applying a derivative operator to them.

Figure 3 shows periodic variance functions generated via Equation (3) with $\sigma=1$ for linear interpolation, linear first and linear second derivative kernels. The linear interpolation is a first-degree member of piecewise, local polynomials. It results in an interpolated signal which is continuous, but its first derivative is discontinuous.

In Figure 4 the generated periodic variance functions (with $\sigma=1$ ) for Catmull-Rom cubic interpolation and its first and second derivative interpolation kernels are illustrated. Cubic interpolation is a very frequently used interpolation technique and has been widely studied. It uses a third-order interpolation polynomial as kernel.

In Figure 5 the variance functions $(\sigma=1)$ for truncated sinc (with 6 supporting points) interpolation, truncated sinc first and second derivative interpolation kernels are shown.

\section{Detection of Periodic Properties of RESAMPLED IMAGES}

In this section we introduce a method capable of detecting the traces of resampling and interpolation using the periodic properties brought into the signal and its derivatives by low order interpolation polynomials. The goal is to determine whether the signal being investigated or some of its portion has been resampled/interpolated or not. In concrete, we pay our attention to individual transformations forming the affine transformation.

The proposed method is based on a few main steps: ROI selection, signal derivative computation, radon transformation and search for periodicity. Each step is explained separately in the following sections.

\section{A. Region of Interest Selection}

In general, a typical image, $f(x, y)$, consists of several consistent regions. To investigate if any of these regions have been resampled we select this region by a block of $R \times R$ pixels (we denote this block by $b(x, y)$ ) and apply the method to this image subset. If it is not possible to define any ROI in the given image or there is a need to find all resampled regions, the image can be tiled by overlapping blocks, $b_{i}(x, y)$, of $R \times R$ pixels. Blocks can be horizontally slid by $N, N \in \mathcal{N}$ pixels rightwards starting with the upper left corner and ending with the bottom right corner. Each block can be analyzed by the method separately. In our experiments $R$ is mostly set to 128 pixels. In other words, we analyze windows of $128 \times 128$ pixels.

\section{B. Signal Derivative Computation}

To emphasize the presence of the periodic properties in the covariance structure of the resampled image, the $n$th derivative of the investigated region, $b(x, y), D^{n}\{b(x, y)\}$, is computed. This is done by applying an approximate derivative operator calculating differences between adjacent pixels of rows of $b(x, y)$. In our experiments the derivative order, $n$, is set to 2 . The used derivative kernel is $[1,-2,1]$. Similar results can be achieved by other derivative orders or using a laplace operator as well as Gabor filters.

\section{Radon Transformation}

To be able to find traces of affine transformation we employ a radon transformation. The radon transformation computes projections of magnitudes of $D^{n}\{b(x, y)\}$ along specified directions determined by angle $\theta$, see Figure 6 . The projection is a line integral in a certain direction. The line integral can be expressed in the following way:

$$
\rho_{D^{n}\{b\}}(x, y)=\int_{L}\left|D^{n}\{b(x, y)\}\right| d l
$$

By assuming that

$$
\left[\begin{array}{l}
x^{\prime} \\
y^{\prime}
\end{array}\right]=\left[\begin{array}{cc}
\cos \theta & \sin \theta \\
-\sin \theta & \cos \theta
\end{array}\right]\left[\begin{array}{l}
x \\
y
\end{array}\right]
$$

it is possible to represent the radon transform the in following way:

$$
\rho_{\theta}\left(x^{\prime}\right)=\int_{-\infty}^{\infty}\left|D^{n}\{b(x, y)\}\right| \cdot\left(x^{\prime} \cos \theta-y^{\prime} \sin \theta, x^{\prime} \sin \theta+y^{\prime} \cos \theta\right) d y^{\prime}
$$

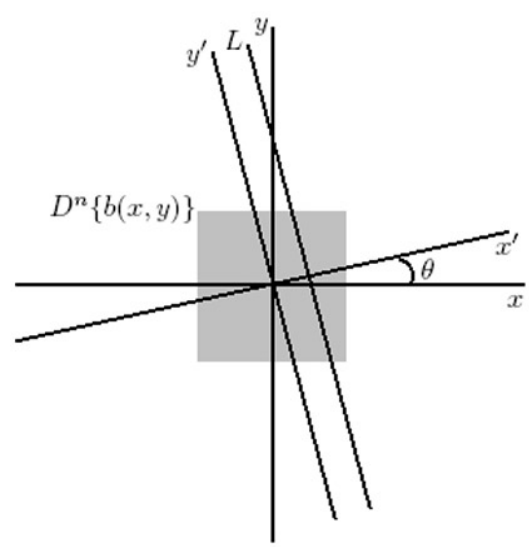

Fig. 6. Radon transformation.

To compute the radon transformation, pixels are divided into four subpixels and each subpixel projected separately. The radon transformation is computed at angles $\theta$ from 0 to $179^{\circ}$, in $1^{\circ}$ increments. Hence, the output of this section is 180 onedimensional vectors, $\rho_{\theta}\left(\theta\right.$ denotes the orientation of $x^{\prime}$ axis counterclockwise from the $x$ axis, see Figure 6). 


\section{Search for Periodicity}

The previous section results in 180 vectors $\rho_{\theta}$. If the investigated region has been resampled, corresponding autocovariance sequences of $\rho_{\theta}$ contain a specific strong periodicity. The autocovariance can be computed in this way:

$$
R_{\rho_{\theta}}(k)=\sum_{i}\left(\rho_{\theta}(i+k)-\overline{\rho_{\theta}}\right)\left(\rho_{\theta}(i)-\overline{\rho_{\theta}}\right)
$$

As mentioned previously, our goal is only to determine if the image being investigated has undergone affine transformation. Hence, we focus only on the strongest periodic patterns present in the autocovariance sections $R_{\rho_{\theta}}$. The effect of this could be that when the analyzed image has undergone several geometric transformations, our method may not detect all particular transformations present in this signal, but only those that have the clearest and strongest periodic properties.

To emphasize and easily detect the periodicity, a derivative filter of order one is applied to vectors $\rho_{\theta}$. After this, in order to easily exhibit strong peaks signifying interpolation, the magnitudes of the Fast Fourier transformation of obtained sequences $R_{\rho_{\theta}}$ are computed. To easily detect the mentioned periodicity, the magnitudes of FFT, $\left|\mathrm{FFT}\left(R_{\rho_{\theta}}\right)\right|$, are all combined and plotted together to create the main output of the method (for example, see Figure 9). As it will be apparent from the next section, if the analyzed region contains interpolation, peaks in the spectrum are very clear and strong and cannot be missed. The spectrum of such a signal has totally different properties of those of non-interpolated signals (see Figures 9 and 10). To automatically detect the interpolation peaks, we apply a simple and strict threshold-based peak detector searching for the local maximum (peaks $n$ times greater than a local average magnitude).

It can be interesting to mention that the presented method can help to estimate the parameters of detected transformation. For example, in the case of rotation, peaks appearing in the spectrum can help us to determine the angle of rotation transformation. Furthermore, as pointed out in [11], when we are concerned with scaling, there is a direct relation between the normalized position of interpolation peaks, $f_{n}$, and the scaling factor, $N$ :

$$
N= \begin{cases}\frac{1}{f_{n}}, & f_{n} \geq 0.5 \\ \frac{1}{1-f_{n}}, & f_{n}<0.5\end{cases}
$$

The method described in this section is always separately applied also to the columns of $b(x, y)$. This is because of the fact that some transformations and images exhibit clearer periodicity in this direction.

So far, we were concerned with TIFF format images. When the method is applied to JPEG compressed images (we assume that the JPEG compression is carried out after the interpolation process), the processing of the method's output must be a little modified. As it is well-known, the JPEG compression technique divides the image into $8 \times 8$ pixel blocks to which is applied a DCT based coding. This blocking artifact brings into the image a periodicity producing peaks at normalized frequencies at positions $1 / 8,2 / 8,3 / 8,5 / 8,6 / 8$ and $7 / 8$ (this can be simply shown by applying the method to

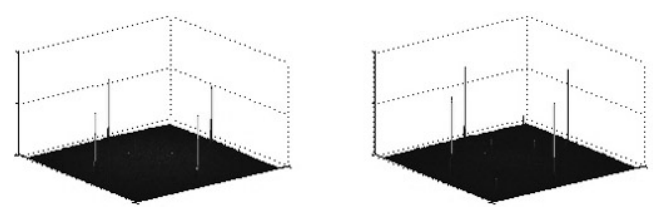

Fig. 7. Bilinear interpolation (interpolation factor 1.7).

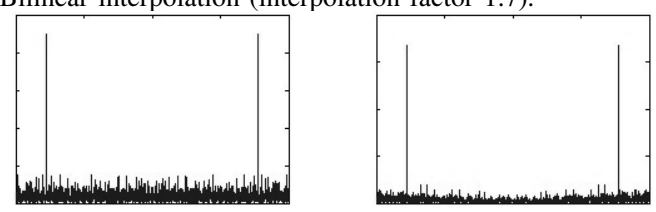

Fig. 8. Cubic interpolation (interpolation factor 0.9).

JPEG compressed images with quality factor 95 and lower). Therefore, when the output of the method is analyzed, these peaks should be ignored. Please note that peaks corresponding to a few resampling factors occur at the same positions as well. Hence, for these factors, it is not possible to make a decision using the presented method's output (for example, scaling factor 0.8 or 1.6 , see Table I).

\section{EXPERIMENTAL RESULTS}

Figure 7 shows a two-dimensional version of the described theory (Equation (7)) for bilinear interpolation with scaling factor $N=1.7$. The entries of the original two-dimensional signal have been chosen randomly from a normal distribution with mean zero and variance one. Figure 8 illustrates that the theory studied in this paper works well for one-dimensional signals as well. Here the theory has been applied to a onedimensional cubic interpolated signal with interpolation factor $N=0.9$. The results for both examples are shown for derivative operator $D^{n}$ with $n=0$ and 1 .

Figure 9 shows the output of the method applied to a resampled region of an investigated image Figure (1). The investigated image in this case consists of two parts, one of which is resized. As it is apparent peaks signifying resampling are strong and clearly detectable. Figure 10 illustrates the case when this method is applied to a non-resampled region. It is apparent the spectrum has totally different properties and there are no strong peaks signifying resampling. The size of the investigated window in both examples is $128 \times 128$ pixels.

Figures 11,12,13 and 14 show outputs of the method applied to several TIFF format images that have undergone various transformations. Shown are outcomes based on rows derivative. The size of the investigated region (denoted by a black box) in all cases is $128 \times 128$ pixels. It is apparent that peaks signifying interpolation are clearly detectable. Figure 15 shows the output of the method applied to a JPEG format interpolated image (with JPEG quality factor 95). The JPEG compression was carried out after the interpolation process. Here, despite the fact that peaks corresponding to JPEG have occurred, the resampling has been successfully recognized. Also here the size of the investigated region is $128 \times 128$ pixels.

In the second part of this section, a quantitative measure of the efficiency of the proposed method is carried out. The method has been applied to 40 images corrupted by 
(a)

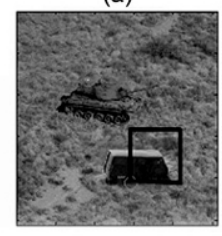

(c)

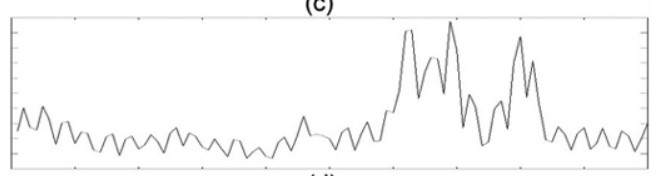

(d)

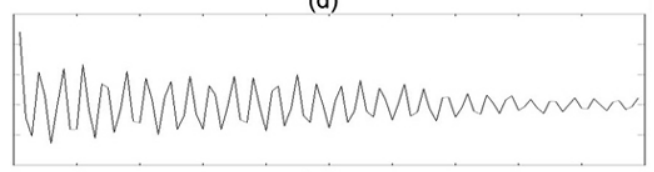

(e)

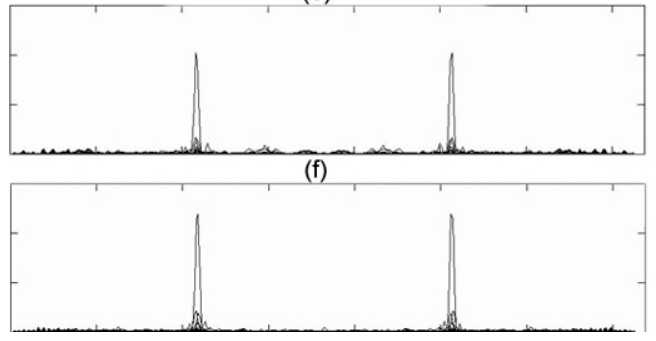

Fig. 9. Shown are: (a) the investigated region $b(x, y)$ (denoted by a black box, $128 \times 128$ pixels); (b) the magnitudes of the rows-based signal derivative $D^{2}\{b(x, y)\}$ (inverted); (c) the radon transformation output $\rho_{\theta}$ for $\theta=90^{\circ}$; (d) the autocovariance $R_{\rho_{\theta}}\left(\theta=90^{\circ}\right)$; (e) the rows-based output of the proposed method ( $\left.\left|\mathrm{FFT}\left(R_{\rho_{\theta}}\right)\right|\right)$; (f) the columns-based output of the method. Peaks are clear and signify interpolation. The radon transformation was computed at angles $\theta$ from 0 to $179^{\circ}$, in $1^{\circ}$ increments. The investigated image is shown in Figure 1.

various transformations. For a few examples of the test images collection see Figure 16. The size of test images was $512 \times 512$ pixels. To do a quantitative measure, the presented method has been applied to the whole image (in other words, the size of investigated region was $512 \times 512$ pixels). In all cases the bicubic interpolation method was used. The method was applied separately to rows and columns of tested images. All experiments were carried out in Matlab.

Specifically, Table I shows the detection accuracy of the method applied to bicubic resized images. The detection accuracy expresses the success of the method in expressing the interpolation by clear and easily detectable peaks, either in row-based or column-based output (for example, see Figure 9 ). Note that the detection is nearly perfect for scaling factors greater than 1.03. Table II shows the detection accuracy of the proposed method applied to rotated images. Table III shows the detection accuracy of the proposed method applied to various skewing factors (skewing was applied to both $\mathrm{x}$ and $\mathrm{y}$ directions). Shown tables contain the detection accuracy of the method in respect to TIFF format images, lossy JPEG format for qualities 95, 97, 100 and white gaussian noise with SNR of 20, 30, 40 and 50. JPEG images were obtained from the non-compressed transformed images. Noisy images were obtained by adding white gaussian noise to the noncompressed transformed images. First, the method has been

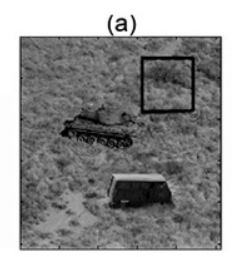

(c)

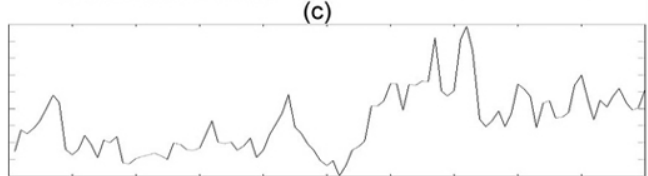

(d)

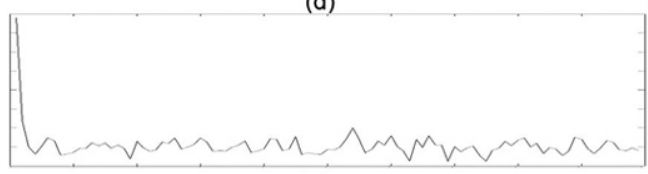

(e)

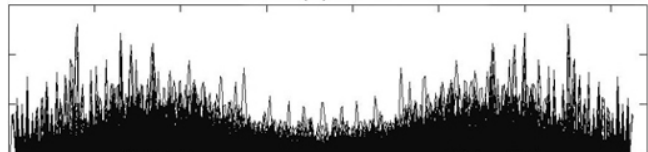

Fig. 10. Shown are: (a) the investigated region $b(x, y)$ (denoted by a black box, $128 \times 128$ pixels); (b) the magnitudes of the rows-based signal derivative $D^{2}\{b(x, y)\}$ (inverted); (c) the radon transformation output $\rho_{\theta}$ for $\theta=90^{\circ}$; (d) the autocovariance $R_{\rho_{\theta}}\left(\theta=90^{\circ}\right)$; (e) the rows-based output of the proposed method $\left(\left|\operatorname{FFT}\left(R_{\rho_{\theta}}\right)\right|\right)$. The radon transformation was computed at angles $\theta$ from 0 to $179^{\circ}$, in $1^{\circ}$ increments. The investigated region has not undergone any geometric transformation. Hence, there are no clear or strong peaks. The spectrum has totally different properties compared to Figure 9. The investigated image is shown in Figure 1.

applied to the rows of tested images. Then the same was applied to the columns of tested images. For correctly detected resized TIFF format images, we tried also to estimate the particular scaling factors using the position of the occurred interpolation peaks. This was carried out for scaling factors greater than 1.03 shown in Table I. The estimation process was based on finding the global maximum in the output of the method. The detection accuracy was near $100 \%$. The same was carried out for rotated TIFF format images (for rotation angles greater than $3^{\circ}$ shown in Table II). Also here, based on the interpolation peaks positions, we tried to estimate the particular rotation angles. The detection accuracy was again near $100 \%$.

During the experimental phase, the method was also applied to the original (non-interpolated) versions of tested images. This resulted in a false positive rate of $12 \%$. Most of the false positives were caused by textures present in these images. Since the method is focused on searching for periodic traces of interpolation, non-interpolated images containing strong textures can yield an output similar to interpolated images. This limitation probably will occur in all methods concerned with the periodic patterns of interpolation. In our method, we prefer to have a higher false positive rate than missing forgery. To strengthen our evidence of forgery, other existing forgery detection methods as well as a texture analyzing step can be employed. 


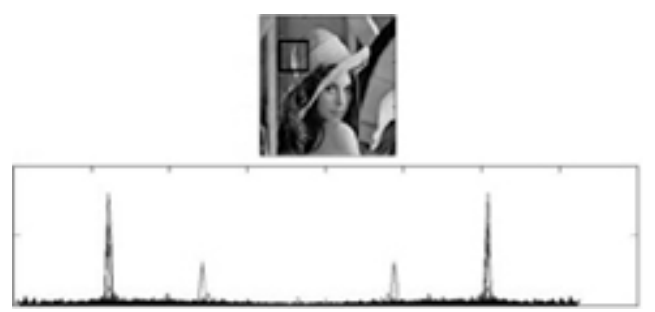

Fig. 11. Scaling factor=1.2 (nearest neighbor interpolation)

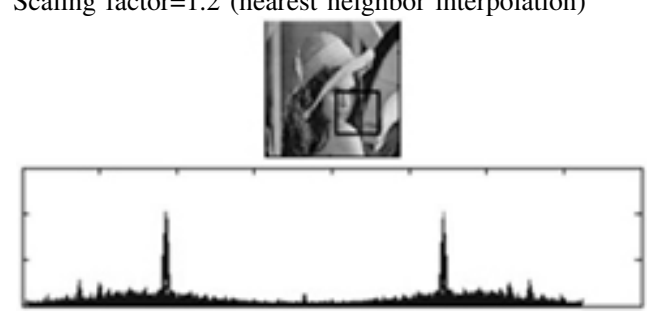

Fig. 12. Scaling factor $=0.8$ (bicubic interpolation)

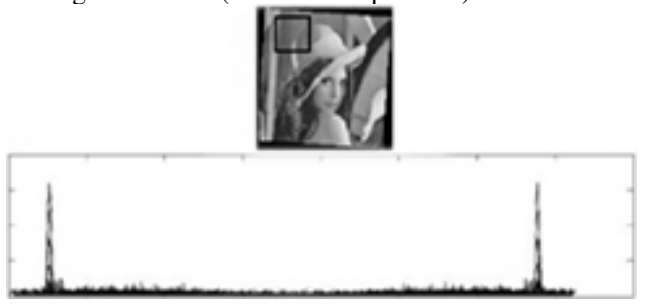

Fig. 13. Rotation $=-4^{\circ}$ (bilinear interpolation)
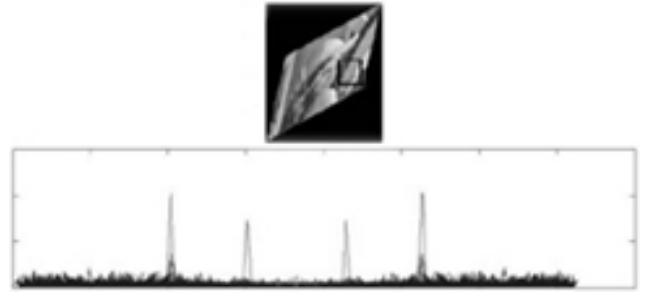

Fig. 14. Skewing factor in $\mathrm{x}$-direction $=-0.4$, in $\mathrm{y}$-direction $=-0.4$ (bilinear interpolation); rotation $=10^{\circ}$ (bilinear interpolation).

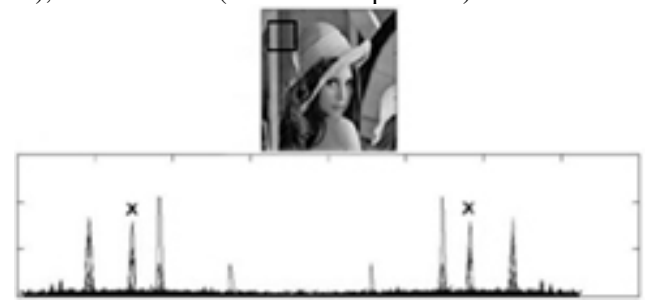

Fig. 15. Scaling factor=1.25 (bicubic interpolation). JPEG format with quality factor 95. Peaks corresponding to the scaling transformation are denoted by $\times$. Other peaks belong to JPEG blocking artifacts.

\section{DISCUSSION}

Obtained results show that the proposed method makes possible in a simple and fast way find traces of general affine transformation when a low order interpolation polynomial has been used.

Please note that not all resampling operations bring detectable changes into the covariance structure of the signal. For instance, the scaling factor 0.5 does not introduce any periodic correlation. This can be easily noticed from its resampling matrix (by resampling matrix we understand the matrix $\mathbf{M}$, where $f^{r}=\mathbf{M} f_{k}$ ):

$$
\mathbf{M}_{\mathbf{0 . 5}}=\left(\begin{array}{cccccc}
1 & 0 & 0 & 0 & 0 & \\
0 & 0 & 1 & 0 & 0 & \\
0 & 0 & 0 & 0 & 1 & \\
& & & & & \ddots
\end{array}\right)
$$

Furthermore, it must be noted that because of aliasing there are resampling factors which have indistinguishable periodic patterns (for example scaling factors 1.5 and 3). For these factors, peaks in the spectrum occur in the same positions. This can be noticed also by analyzing the resampling matrix of these interpolation factors. Hence, the presented method itself cannot propose uniqueness in the estimating of resampling factors.

The proposed method works well for low order interpolation polynomials: nearest neighbor, linear or cubic. These interpolators have a strong detectable effect on the covariance structure of the signal. The detection performance decreases as the order of interpolation polynomial increases. Different interpolation orders introduce correlations of varying degrees between neighboring samples. These correlations become more difficult to detect as each interpolated sample value is obtained as a function of more samples. For example, in an image interpolated by a truncated sinc with $N=9$, interpolated pixels are functions of 81 pixels. Note that when the ideal sinc interpolator is used, the covariance structure of the signal does not change and therefore this interpolator is not detectable. This statement can be shown be evaluating Equation (3) for sinc, which gives 1 for all $x$ (it is due to the fact that $\left.\sum_{k=-\infty}^{\infty} \mathcal{D}^{n}\{\operatorname{sinc}\}(x-k)^{2}=1\right)$.

It must be mentioned that results obtained can be affected by the presence of other correlations in the signal. Hence, the best results are obtained by applying the method to interpolated white noise signal (the autocorrelation of a white noise signal has a strong peak at $x=0$ and is close to 0 elsewhere).

The proposed method is based on a statistical approach. It could be said that larger investigated regions give stronger and more accurate results. Our experiments show that generally the smallest acceptable size of investigated regions is $128 \times 128$ pixels. But we have to mention that, for instance, many resampling operations can be recognized in a smaller investigation window $(64 \times 64$ pixels $)$ as well. On the other hand, some strong skewing factors need a larger investigation window. It depends on the signal spatial correlation and distribution of image pixels.

By applying the method to JPEG compressed images (when JPEG compression is carried out after the interpolation process), the detection performance decreases. JPEG is a lossy compression format. It brings noise into the image. Experiments show that the presented method works well for JPEG compression quality of 95 - 100. But, generally, the results obtained are based on image properties. By adding noise to the signal the interpolation-based pixels correlation becomes corrupted and difficult to detect.

In this work we were concerned with gray-level images. There are several ways to use the presented method for RGB 
TABLE I

DETECTION ACCURACY [\%] AS A FUNCTION OF DIFFERENT SCALING FACTORS, TIFF, JPEG COMPRESSION QUALITIES AND SIGNAL-TO-NOISE RATIOS. EACH CELL CORRESPONDS TO THE AVERAGE DETECTION ACCURACY FROM 40 IMAGES.

\begin{tabular}{|l||c|c|c|c|c|c|c|c|c|c|c|}
\hline scaling factor & 0.55 & 0.60 & 0.65 & 0.70 & 0.75 & 0.80 & 0.85 & 0.90 & 0.95 & 0.97 & 0.99 \\
\hline TIFF & 67 & 82 & 92 & 95 & 97 & 100 & 100 & 100 & 100 & 90 & 35 \\
\hline JPEG 100 & 67 & 82 & 92 & 95 & 97 & 0 & 100 & 100 & 100 & 90 & 35 \\
\hline JPEG 97 & 55 & 67 & 85 & 92 & 95 & 0 & 100 & 100 & 100 & 87 & 30 \\
\hline JPEG 95 & 42 & 55 & 70 & 77 & 85 & 0 & 87 & 90 & 85 & 57 & 12 \\
\hline SNR 50 dB & 67 & 82 & 92 & 95 & 97 & 100 & 100 & 100 & 100 & 90 & 32 \\
\hline SNR 40 dB & 60 & 80 & 90 & 92 & 97 & 100 & 100 & 100 & 100 & 87 & 25 \\
\hline SNR 30 dB & 55 & 72 & 85 & 90 & 92 & 95 & 95 & 95 & 87 & 47 & 10 \\
\hline SNR 20 dB & 5 & 5 & 7 & 10 & 10 & 10 & 12 & 12 & 5 & 5 & 0 \\
\hline \hline scaling factor & 1.01 & 1.03 & 1.05 & 1.10 & 1.15 & 1.20 & 1.25 & 1.30 & 1.35 & 1.40 & 1.45 \\
\hline TIFF & 35 & 90 & 100 & 100 & 100 & 100 & 100 & 100 & 100 & 100 & 100 \\
\hline JPEG 100 & 35 & 90 & 100 & 100 & 100 & 100 & 100 & 100 & 100 & 100 & 100 \\
\hline JPEG 97 & 30 & 87 & 100 & 100 & 100 & 100 & 100 & 100 & 100 & 100 & 100 \\
\hline JPEG 95 & 12 & 57 & 87 & 95 & 95 & 100 & 100 & 100 & 100 & 100 & 100 \\
\hline SNR 50 dB & 32 & 90 & 100 & 100 & 100 & 100 & 100 & 100 & 100 & 100 & 100 \\
\hline SNR 40 dB & 25 & 87 & 100 & 100 & 100 & 100 & 100 & 100 & 100 & 100 & 100 \\
\hline SNR 30 dB & 12 & 47 & 92 & 97 & 97 & 100 & 100 & 100 & 100 & 100 & 100 \\
\hline SNR 20 dB & 0 & 7 & 7 & 12 & 15 & 17 & 20 & 20 & 25 & 27 & 30 \\
\hline \hline Scaling factor & 1.50 & 1.55 & 1.60 & 1.65 & 1.70 & 1.75 & 1.80 & 1.85 & 1.95 & 2.05 & 2.10 \\
\hline TIFF & 100 & 100 & 100 & 100 & 100 & 100 & 100 & 100 & 100 & 100 & 100 \\
\hline JPEG 100 & 100 & 100 & 0 & 100 & 100 & 100 & 100 & 100 & 100 & 100 & 100 \\
\hline JPEG 97 & 100 & 100 & 0 & 100 & 100 & 100 & 100 & 100 & 100 & 100 & 100 \\
\hline JPEG 95 & 100 & 100 & 0 & 100 & 100 & 100 & 100 & 100 & 100 & 100 & 100 \\
\hline SNR 50 dB & 100 & 100 & 100 & 100 & 100 & 100 & 100 & 100 & 100 & 100 & 100 \\
\hline SNR 40 dB & 100 & 100 & 100 & 100 & 100 & 100 & 100 & 100 & 100 & 100 & 100 \\
\hline SNR 30 dB & 100 & 100 & 100 & 100 & 100 & 100 & 100 & 100 & 100 & 100 & 100 \\
\hline SNR 20 dB & 30 & 30 & 30 & 30 & 30 & 30 & 30 & 30 & 30 & 30 & 30 \\
\hline
\end{tabular}

TABLE II

DETECTION ACCURACY [\%] AS A FUNCTION OF DIFFERENT ROTATION ANGLES, TIFF, JPEG COMPRESSION QUALITIES AND SIGNAL-TO-NOISE RATIOS. EACH CELL CORRESPONDS TO THE AVERAGE DETECTION ACCURACY FROM 40 IMAGES.

\begin{tabular}{|l||c|c|c|c|c|c|c|c|}
\hline rotation angle & $1^{\circ}$ & $3^{\circ}$ & $5^{\circ}$ & $10^{\circ}$ & $15^{\circ}$ & $20^{\circ}$ & $30^{\circ}$ & $40^{\circ}$ \\
\hline TIFF & 22 & 85 & 100 & 100 & 100 & 100 & 100 & 100 \\
\hline JPEG 100 & 22 & 85 & 100 & 100 & 100 & 100 & 100 & 100 \\
\hline JPEG 97 & 17 & 77 & 100 & 100 & 100 & 100 & 97 & 92 \\
\hline JPEG 95 & 7 & 57 & 92 & 100 & 100 & 97 & 85 & 70 \\
\hline SNR 50 dB & 22 & 85 & 100 & 100 & 100 & 100 & 100 & 100 \\
\hline SNR 40 dB & 17 & 80 & 100 & 100 & 100 & 100 & 100 & 100 \\
\hline SNR 30 dB & 10 & 52 & 90 & 100 & 100 & 100 & 87 & 90 \\
\hline SNR 20 dB & 0 & 5 & 12 & 20 & 22 & 25 & 12 & 10 \\
\hline
\end{tabular}

TABLE III

DETECTION ACCURACY [\%] AS A FUNCTION OF DIFFERENT SKEWING FACTORS, TIFF AND SIGNAL-TO-NOISE RATIO 40 DB. EACH CELL CORRESPONDS TO THE AVERAGE DETECTION ACCURACY FROM 40 IMAGES.

\begin{tabular}{|l||c|c|c|c|c|c|c|c|c|c|}
\hline skewing factor & 0.01 & 0.03 & 0.05 & 0.10 & 0.15 & 0.20 & 0.25 & 0.30 & 0.35 & 0.40 \\
\hline TIFF & 5 & 90 & 97 & 100 & 100 & 100 & 100 & 100 & 100 & 100 \\
\hline SNR 40 dB & 0 & 0 & 0 & 0 & 0 & 0 & 0 & 0 & 0 & 0 \\
\hline
\end{tabular}

images. For instance, the method can be applied to each channel separately.

\section{CONCLUSION}

In this paper we have analyzed specific periodic properties present in the covariance structure of interpolated signals and their derivatives. Furthermore, we have introduced a brief twodimensional extension of the described theory. Also we have analyzed an application of Taylor series to the interpolated signals. The main contribution of the paper is a method capable of easily detecting traces of scaling, rotation, skewing transformations and any of their arbitrary combinations. The method is fast, blind and efficient. It works for wide variety of resampling factors. Another advantage of the method is that it can be very helpful in the estimation of scaling factors or rotation angles.

We believe that the theory studied here can be useful in image security and authentication as well as in applications based on statistical approaches using interpolated signals.

\section{REFERENCES}

[1] E. Meijering, "A chronology of interpolation: From ancient astronomy to modern signal and image processing," Proceedings of the IEEE, vol. 90, no. 3, pp. 319-342, March 2002.

[2] M. M. Yeung, "Digital watermarking." Commun. ACM, vol. 41, no. 7, pp. 30-33, 1998.

[3] C. Rey and J.-L. Dugelay, "A survey of watermarking algorithms for image authentication," EURASIP Journal on applied Signal Processing Volume 2002 N6 - June 2002, special issue on image analysis for multimedia interactive services, pp. 613-621, 2002. 


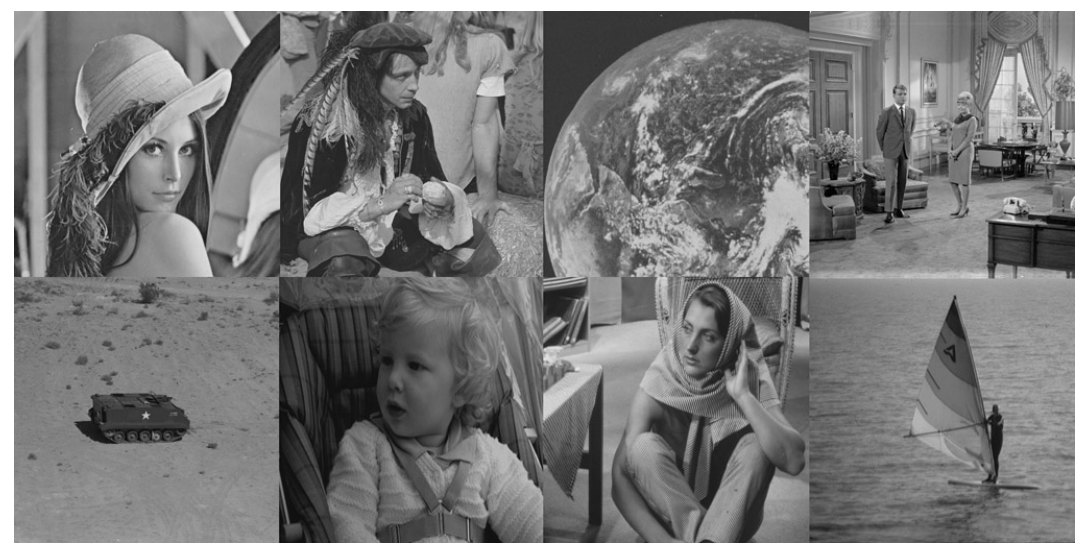

Fig. 16. A few examples of test images.

[4] J. Fridrich, "Methods for tamper detection in digital images," Proceedings of Multimedia and Security Workshop at ACM Multimedia, pp. 19-23.

[5] B. Mahdian and S. Saic, "Detection of copy-move forgery using a method based on blur moment invariants," Forensic science international, vol. 171, no. 2-3, pp. 180-189, 2007.

[6] J. Fridrich, D. Soukal, and J. Lukas, "Detection of copy-move forgery in digital images," in Proceedings of Digital Forensic Research Workshop. Cleveland, OH, USA: IEEE Computer Society, August 2003, pp. 55-61.

[7] A. Popescu and H. Farid, "Exposing digital forgeries by detecting traces of re-sampling," IEEE Transactions on Signal Processing, vol. 53, no. 2, pp. 758-767, 2005.

[8] M. Johnson and H. Farid, "Exposing digital forgeries by detecting inconsistencies in lighting," in ACM Multimedia and Security Workshop, New York, NY, 2005, pp. 1-9.

[9] A. Popescu and H. Farid, "Statistical tools for digital forensics," in 6th International Workshop on Information Hiding, Toronto, Cananda, 2004, pp. 128-147.

[10] S. Prasad and K. R. Ramakrishnan, "On resampling detection and its application to image tampering," in Proceedings of the IEEE International Conference on Multimedia and Exposition, Toronto, Canada, 2006, pp. $1325-1328$.

[11] A. C. Gallagher, "Detection of linear and cubic interpolation in jpeg compressed images," in CRV '05: Proceedings of the The 2nd Canadian Conference on Computer and Robot Vision (CRV'05). Washington, DC, USA: IEEE Computer Society, 2005, pp. 65-72.

[12] J. A. Parker, R. V. Kenyon, and D. E. Troxel, "Comparison of interpolation methods for image resampling," IEEE Transactions on Medical Imaging, vol. 2, no. 1, pp. 31-39, 1983.

[13] H. Hou and H. Andrews, "Cubic splines for image interpolation and digital filtering," IEEE Transactions on Acoustics, Speech and Signal Processing, vol. 26, no. 6, pp. 508-517, 1978.

[14] E. H. W. Meijering, W. J. Niessen, and M. A. Viergever, "Piecewise polynomial kernels for image interpolation: A generalization of cubic convolution." in ICIP (3), 1999, pp. 647-651.

[15] G. Rohde, C. Berenstein, and D. Healy, "Measuring image similarity in the presence of noise," Proceedings of the SPIE Medical Imaging: Image Processing, vol. 5747, pp. 132-143, February 2005.

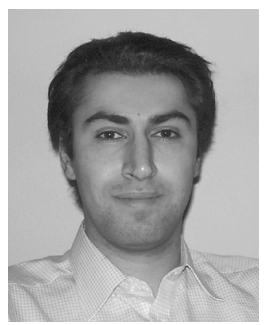

Babak Mahdian received the M.Sc. degree in Informatics and Computer Engineering from the University of West Bohemia, Pilsen, Czech Republic, in 2004. He is currently a Ph.D. candidate in Mathematical Engineering at the Czech Technical University, Prague, Czech Republic. His current research interests include all aspects of digital image processing and pattern recognition, particularly digital image authentication, interpolation theory, facial expressions recognition and multimodal HCI.

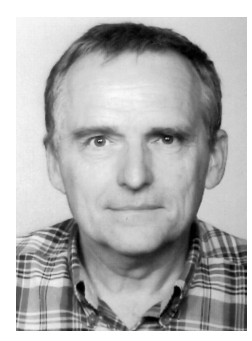

Stanislav Saic received the M.Sc. degree in Physical Electronics from the Czech Technical University, Prague, Czech Republic, in 1973, and the CSc. degree (corresponds to Ph.D. degree) in Radioelectronics from the Czechoslovak Academy of Sciences, Prague, Czech Republic, in 1980. Since 1973, he has been with the Institute of Information Theory and Automation, Academy of Sciences of the Czech Republic, Prague, where he held the position of Head of the Department of Image Processing in 1985 - 1994. His current research interests include all aspects of digital image and signal processing, particularly Fourier transform, image filters, remote sensing and geosciences. 